\title{
FUNCIONAMIENTO DE LAS MIRAS NOCTURNAS PARA EL FUSIL GALIL DE INDUMIL
}

\section{OPERATION OF NIGHT SIGHTS FOR THE GALIL RIFLE PRODUCED BY INDUMIL}

\author{
Ernesto, Villarreal Silva \\ Ingeniero Nuclear, M.S., Ph.D., Director, Ingeniería en Multimedia, Facultad de Ingeniería \\ Universidad Militar Nueva Granada, Bogota, Colombia, evillar@umng.edu.co
}

Fecha de recepción: 17septiembre de 2007.

Fecha de aprobación: 16 de junio de 2008.

\section{RESUMEN}

El fusil Galil fue desarrollado por la Industria Militar Israelí y posteriormente fabricado en otros países. En Colombia el fusil es fabricado por la industria militar (INDUMIL) en cantidades crecientes, tanto para exportación como para el mercado local. El rifle es ahora el estándar de todas las fuerzas armadas colombianas. Con el paso de los años INDUMIL ha podido fabricar un mayor porcentaje de los componentes necesarios para construir el rifle pero hay algunas que aun se compran. Como las miras nocturnas que permiten apuntar el rifle en la noche en la dirección correcta; estas usan luces de Tritio que brillan en la noche. La Universidad Militar Nueva Granada ha iniciado un proyecto conjunto con INDUMIL para desarrollar y ensamblar las miras en la institución. Este artículo describe las bases teóricas del funcionamiento de las luces de Tritio.

Palabras clave: Luces de Tritio, Miras nocturnas, Fosforescencia, Tritio.

\section{ABSTRACT}

The GALIL rifle was developed by the Israeli Military Industries and later on produced in other countries like Colombia. In this country the rifle is made by the military industry (INDUMIL) in increasing numbers, both for export and for the local market. The rifle has become the standard for all Colombian forces. Through the years INDUMIL has been able to manufacture an increasing percentage of the components needed to build the rifle but there are some that are still purchased. One of the pieces acquired is the night sights to be 
able to aim the rifle in the right direction in the dark; they use Tritium lights that glow at night. The Nueva Granada Military University began a joint project with INDUMIL to develop and assemble the sights in the institution. In this writing the theory behind the operation of the Tritium lights is dealt with.

Key words: Tritium lights, Night sights, Phosphorescence, Tritium.

\section{INTRODUCCIÓN}

Las Fuerzas Armadas de Colombia utilizan como estándar el fusil GALIL 5.65 mm versión AR fabricado por INDUMIL. La producción de los componentes del fusil se efectúa en el país con la excepción de algunos elementos como las miras nocturnas. Para lograr la independencia total en la fabricación de las miras nocturnas se ha iniciado un proyecto que permitirá en el futuro cercano construir estas miras en INDUMIL, reemplazando las que hoy día son adquiridas para dotar a más de 40.000 fusiles que se producen anualmente, para venta local y para exportación.

\section{PRELIMINARES}

\subsection{FUNCIONAMIENTO DE LAS MIRAS NOCTURNAS.}

El fusil lleva instalada una mira diurna y una nocturna, que se pueden doblar cuando no están en uso. Cada una de ellas a su vez está compuesta de dos partes: una mira trasera y una delantera como se indica en la Figura 1.

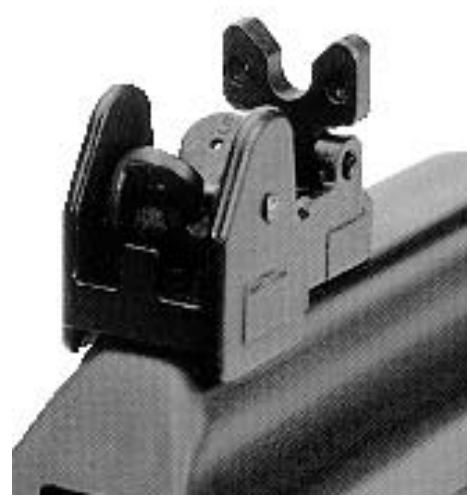

Figura 1a. Mira trasera

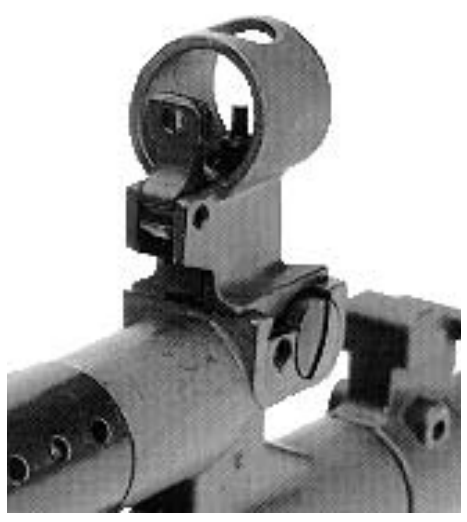

Figura 1b. Mira delantera 


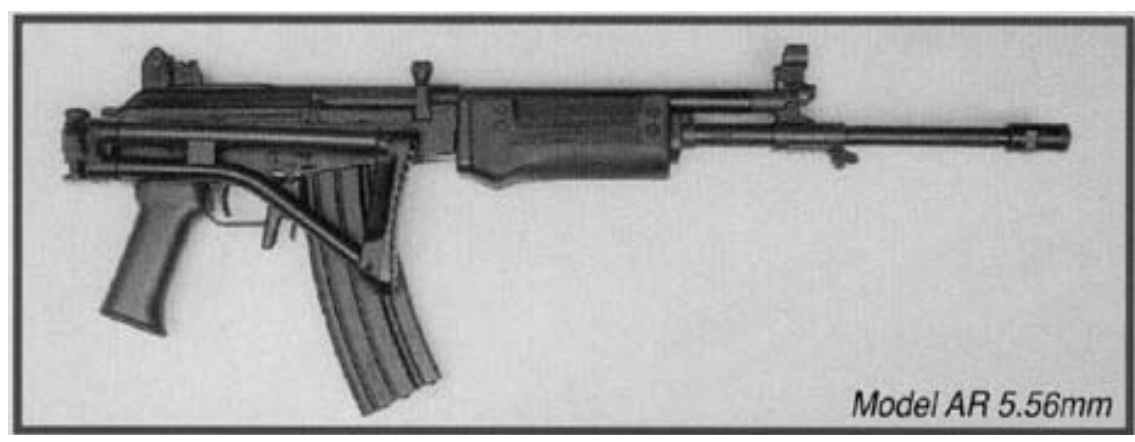

Figura 1c. Fusil Galil [2].

Fuente: [2] http://www.indumil.gov.co/frameCategoria.asp?ldCat=16\&ldCatmostrar=16,

[5] http://www.remtek.com/arms/imi/galil/index.htm

Para hacer uso de la mira nocturna se doblan las partes de la mira diurna que no se necesitan. La mira nocturna posee fuentes de luz verde (dos la mira trasera y una la delantera) lo cual permite apuntar el arma en la dirección deseada. Las fuentes de luz son permanentes pues utilizan el fenómeno de la fosforescencia, es decir de la emisión sostenida de luz, aun en la oscuridad. Para ello hacen uso del material radiactivo Tritio y de un material fosforescente.

\subsection{EL TRITIO}

El Hidrógeno natural está compuesto por dos tipos de átomos, variedades isotópicas que se conocen con los nombres de Protio y Deuterio, quienes se presentan en las proporciones de $99,9844 \%$ y $0,0156 \%$ respectivamente. De otra parte hay una tercera variedad isotópica del Hidrogeno, extremadamente escasa llamada Tritio que se fabrica en la atmósfera permanentemente. El Tritio es la única de las tres variedades cuyos átomos son inestables y se desintegran mediante la emisión de radiactividad, lo que les permite perder energía. Los núcleos de los átomos de Tritio se desintegran mediante la emisión de radiación beta negativa, es decir electrones negativos. La energía de estas partículas emitidas es inferior a $18,6 \mathrm{keV}$, en promedio $5,7 \mathrm{keV}$, tan baja que esa radiación no puede atravesar una pared de vidrio, por delgada que ésta sea.

La siguiente ecuación indica que cuando el núcleo del átomo de Tritio emite un electrón negativo, se convierte en un núcleo del elemento Helio.

$$
{ }_{1}^{3} \mathrm{H} \rightarrow{ }_{2}^{3} \mathrm{He}+{ }_{-1}^{0} \text { electrón }\left(E_{\max }=18,6 \mathrm{keV}\right)+{ }_{0}^{0} \text { neutrino }
$$

Con el tiempo la cantidad de Tritio presente va disminuyendo de acuerdo con la ley de desintegración radiactiva, de forma exponencial como se muestra en la ecuación 1 y en la Figura 2. 


$$
N(t)=N_{0} e^{\lambda t}(1)
$$

Donde: $\mathrm{N}(\mathrm{t})$ = es la cantidad de átomos de Tritio en el tiempo $t, \mathrm{~N}_{0}=$ es la cantidad inicial de átomos de Tritio,

$\lambda=$ probabilidad de que un átomo se transforme en otro por segundo, y $\mathrm{t}=\mathrm{es}$ el tiempo transcurrido.

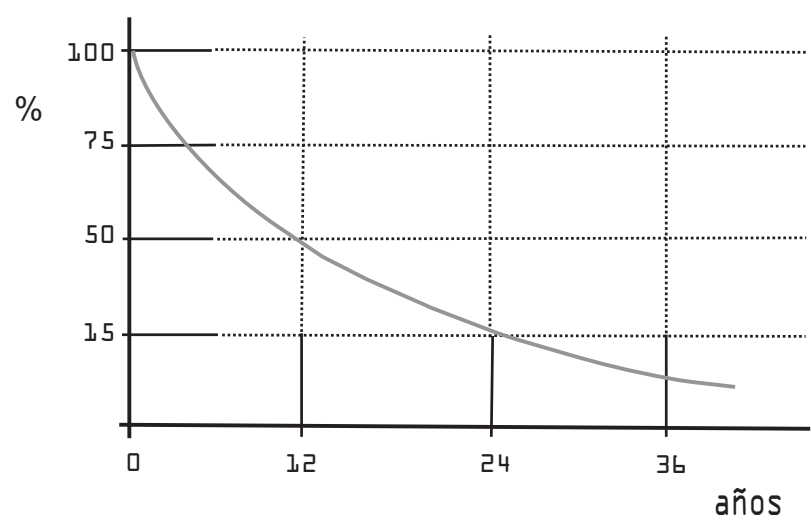

Figura 2. Desintegración radiactiva del Tritio

La Vida media, es decir el tiempo requerido para que la cantidad de Tritio se reduzca a la mitad, es de 12,26 años. Al cabo de dos Vidas medias, 24,5 años, la cantidad de Tritio se habrá reducido a la cuarta parte. Al cabo de tres Vidas medias quedará la octava parte y así sucesivamente, como se muestra en la Figura 2.

El Tritio se fabrica permanentemente en la atmósfera debido a interacciones entre los rayos cósmicos provenientes de estrellas como el Sol y los núcleos de átomos de Nitrógeno presentes en la atmósfera, a través de la reacción 2.

$$
{ }_{7}^{14} \mathrm{~N}+{ }_{0}^{1} \mathrm{n} \rightarrow{ }_{6}^{12} \mathrm{C}+{ }_{1}^{3} \mathrm{H}
$$

Sin embargo la cantidad producida es muy pequeña, lo que resulta en que la concentración de Tritio en la atmósfera sea muy baja. Existen varios procesos para fabricar Tritio en el mundo, por ejemplo irradiando Litio (activación neutrónica) en reactores experimentales, a través de la reacción 3.

$$
{ }_{3}^{6} \mathrm{Li}+{ }_{0}^{1} \mathrm{n} \rightarrow{ }_{2}^{4} \mathrm{He}+{ }_{1}^{3} \mathrm{H}
$$

u obteniéndolo como subproducto del funcionamiento de reactores de agua pesada. En este caso el Tritio se produce cuando el Deuterio presente en las moléculas de agua del refrigerante, captura un neutrón, a través de la reacción 4.

$$
{ }_{1}^{2} \mathrm{H}+{ }_{0}^{1} \mathrm{n} \rightarrow{ }_{1}^{3} \mathrm{H}
$$

El Tritio se produce especialmente para hacer más efectivas las bombas atómicas de fisión nuclear, pues debido a su Vida media relativamente corta, este material debe ser reemplazado periódicamente para que no pierdan su capacidad. De otra parte cantidades pequeñas de Tritio se usan por ejemplo para la construcción de miras nocturnas para armas y en aplicaciones pacificas como señales luminosas para la industria, como se 
explica más adelante. El comercio del Tritio no es libre pues a causa de sus implicaciones bélicas las autoridades nucleares de los países regulan aspectos como la importación, transporte, manipulación y exportación del mismo.

En relación con los materiales radiactivos se define la Actividad como el número de átomos de un radionúclido que se transforma en otro por segundo, así:

$$
\text { Actividad }=\lambda \mathrm{N}(\mathrm{t})
$$

En el caso del Tritio será el número de electrones negativos liberados por núcleos de Tritio en un segundo para transformarse en ${ }^{3} \mathrm{He}$. La Actividad se mide en Becquerelios $(\mathrm{Bq})$, donde un $\mathrm{Bq}$ equivale a la transformación de un átomo por segundo. Por ejemplo, si a partir de cierta cantidad de Tritio observamos que se emiten mil millones de electrones negativos por segundo su Actividad será de un Gigabecquerelio (GBq).

La Actividad es directamente proporcional a la cantidad de material radiactivo presente y disminuye con el tiempo de manera exponencial, como se indica en la fórmula 6:

$$
\text { Actividad }=\lambda N(\mathrm{t})=\lambda \mathrm{N}_{0} \mathrm{e}^{-\lambda \mathrm{t}},
$$

Donde:

$\lambda \mathrm{N}_{0=}$ Actividad inicial.

\subsection{LA FOSFORESCENCIA}

De acuerdo con el modelo simplificado de Bohr (Figura 3) los átomos son como pequeñísimos sistemas solares en cuyo centro esta el núcleo que contiene neutrones y protones y a su alrededor giran electrones. Una diferencia importante entre los átomos y el sistema solar es que mientras en este cada planeta tiene su propia órbita, en los átomos cabe más de un electrón por órbita. La órbita mas cercana al núcleo se llama la órbita K, la siguiente la $\mathrm{L}$, luego viene la $\mathrm{M}$ y así sucesivamente. La cantidad de electrones que caben en una órbita viene dada por la formula $2 q^{2}$ donde q vale 1 para la órbita K, 2 para la órbita L, 3 para la órbita M y así sucesivamente. Por lo tanto en la órbita $K$ caben dos electrones, en la L ocho, etc.

Los átomos de cada elemento tienen un conjunto de órbitas muy propio que los diferencia de los demás. En los átomos de elementos livianos las órbitas están más cerca que en los átomos de elementos pesados.

Debido a la presencia de protones en el núcleo estos son positivos, mientras que los electrones son negativos. La segunda fuerza fundamental, la electromagnética, nos dice 
que los electrones son atraídos por el núcleo, con mayor fuerza entre más cerca estén a él. Por esta razón se asocian con las órbitas de los electrones unos niveles energéticos ( $\mathrm{n}=$ $1,2,3 . .$.$) que nos indican la energía necesaria para que el electrón se pueda desprender$ del núcleo.

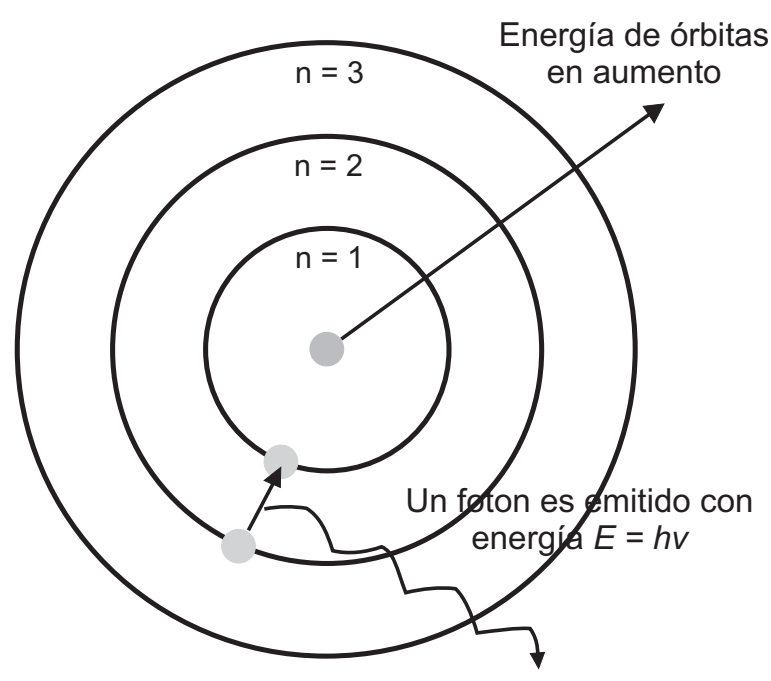

Figura 3. Modelo de Bohr [3].

Fuente: http://es.wikipedia.org/wiki/Modelo_at\% C3\%B3mico de Bohr [3]

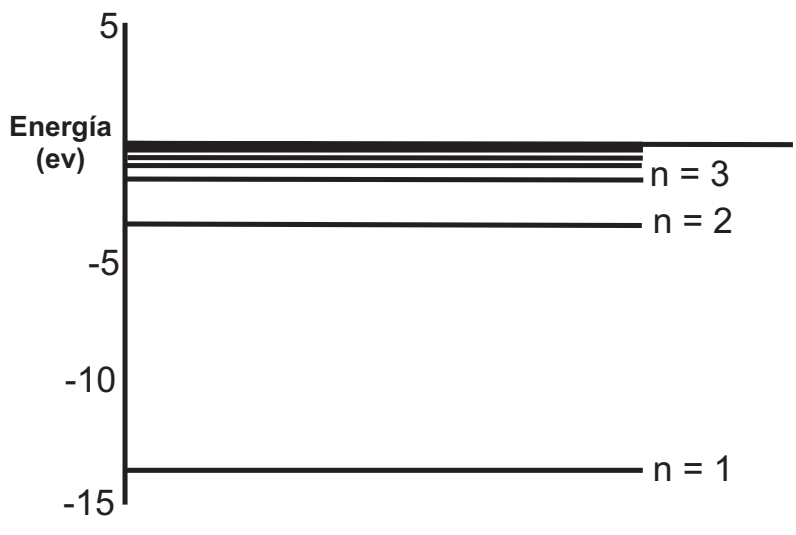

Figura 4. Niveles energéticos asociados con las órbitas del átomo de Hidrogeno

Las Figuras 3 y 4 muestran los niveles energéticos asociados con las órbitas que puede ocupar el único electrón que posee el átomo de Hidrógeno.

Para que un electrón pueda alejarse del núcleo pasando de una órbita cercana al a una alejada, requiere que se le adicione energía pues pasa de una posición en la que está muy atado al núcleo a una en que está menos atado. Por el contrario, cuando un electrón pasa de una órbita alejada del núcleo a una más cercana a él, pierde energía. Esta energía perdida se emite por parte del átomo como un fotón cuya energía corresponde a la diferencia entre las energías asociadas con los niveles energéticos inicial y final de las órbitas entre las cuales se desplaza el electrón. Como se ha dicho, el conjunto de órbitas de los átomos de cada elemento es propio de el y por lo tanto los niveles energéticos asociados con tales órbitas, por lo que los fotones emitidos a partir de átomos de un elemento lo caracterizan de manera inequívoca (Figura 5b). Es decir el espectro de energías de los fotones emitidos es propio de cada elemento. Si uno puede medir la energía del fotón proveniente de un átomo puede averiguar a qué elemento corresponde el mismo. La Figura 5 a muestra como a partir del átomo de Hidrógeno se generan fotones de color azul, rojo y verde, entre otros. 


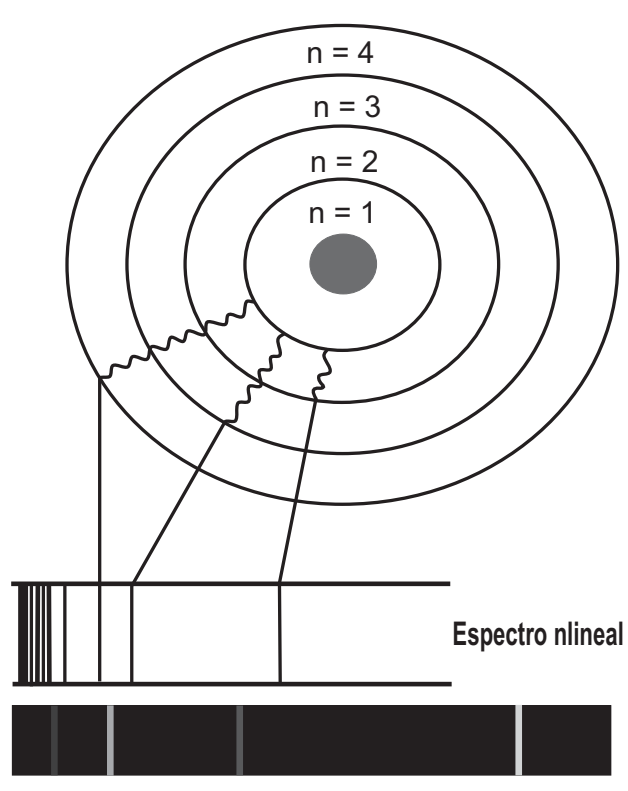

Figura 5a. Emisión de colores del Hidrogeno. Fuente: ZUMDAHL, Steven 2004 [7].

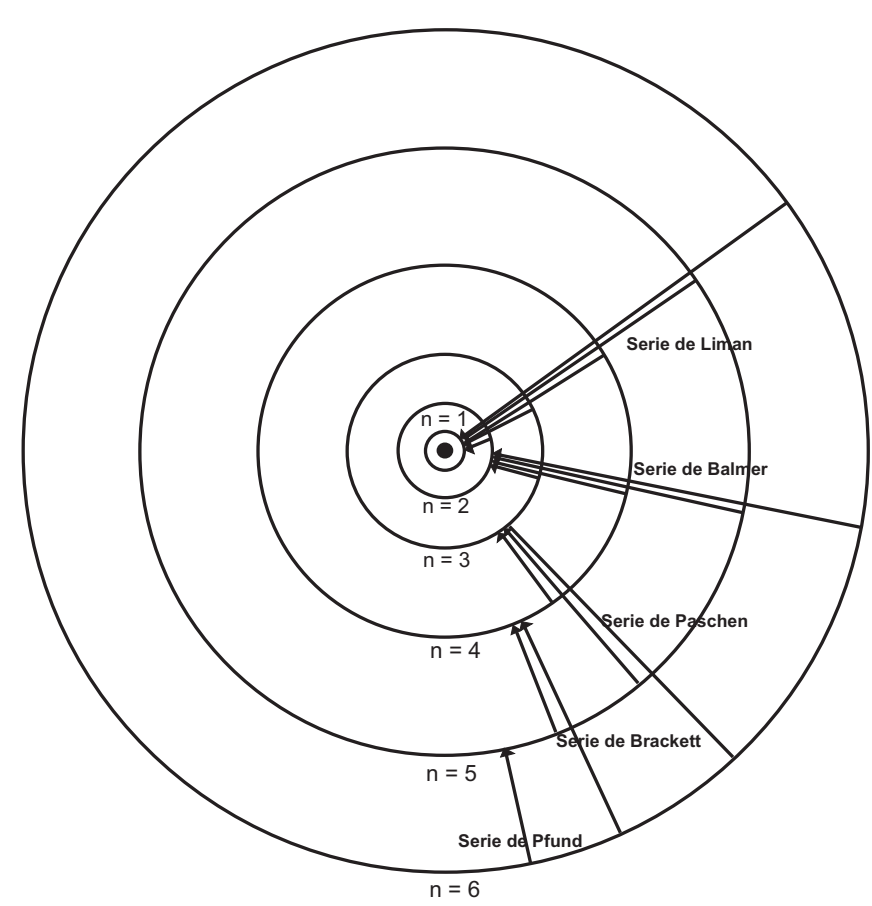

Figura 5b. Espectro del Hidrógeno Fuente: EMERY, Bob [1].

Una ley natural exige a los átomos estar en el estado de mayor estabilidad que les sea posible. Esto significa que los electrones en los átomos llenan las órbitas bajas antes que las altas, pues de esta manera están mas atados al núcleo dando mayor estabilidad al átomo.

Si sobre un átomo llegan fotones de luz o partículas tales como electrones, con energías que coinciden con las diferencias de energía entre niveles energéticos existentes en el átomo, la energía que ellos traen se absorbe por el átomo. Esta energía se usa para desplazar electrones en el átomo entre las órbitas (baja a alta) a que corresponden los niveles energéticos. Este proceso se conoce como excitación de los átomos. Por lo anterior se dice que de la misma manera que los átomos de un elemento tienen un espectro de emisión, tienen un espectro de absorción.

Posterior al proceso de excitación y en desarrollo de la ley natural mencionada anteriormente, electrones de órbitas externas caen para llenar los vacíos dejados por los electrones desplazados en las órbitas bajas; en este proceso pierden energía, la que es liberada a través de diversos procesos, en ocasiones en la forma de un fotón. La energía del mismo depende de la diferencia entre los niveles energéticos de las órbitas entre las que se desplaza el electrón. 
Este desplazamiento de electrones entre órbitas alejadas del núcleo a órbitas cercanas a él produce fotones cuyas energías pueden corresponder a la de los Rayos X, ultravioleta, luz visible, infrarrojo, microondas, etc. La liberación de estos fotones puede ocurrir de manera espontánea (diodos emisores de luz, lámparas de neón) o inducida (láser).

El fenómeno de la fluorescencia consiste en que hay átomos, que emiten fotones de energías correspondientes a la luz visible cuando son iluminados por radiaciones más energéticas. La razón de que la energía de los fotones visibles emitidos sea menor que la de los fotones o partículas incidentes es que el electrón excitado puede perder la energía adquirida a través de diversos procesos, además de la emisión de fotones o se puede pensar que para llenar el vacío dejado por el electrón que es excitado, varios electrones se desplazan secuencialmente. Por ejemplo, si a causa de un fotón incidente un electrón fue desplazado de la órbita uno a la cinco, puede ocurrir que un electrón de la órbita dos caiga a la uno, un electrón de la órbita tres caiga a la dos y un electrón de la órbita cinco caiga a la tres. De esta forma el átomo emitirá varios fotones de energía inferior a la que traía el fotón o partícula incidente. Un buen ejemplo son las lámparas fluorescentes, en las cuales la radiación ultravioleta produce luz visible.

Cuando la emisión de esta luz se produce de forma retardada, el fenómeno recibe el nombre de fosforescencia. Para realzar este efecto se adicionan elementos activadores que aumentan el tiempo durante el cual se produce la emisión de luz. El mecanismo utilizado en los tubos de rayos catódicos consiste en excitar los átomos de compuestos o sustancias fosforescentes, a las que se le han agregado activadores, mediante el bombardeo con partículas subatómicas, electrones.

Se pueden fabricar diferentes materiales fosforescentes que tienen la capacidad de emitir luz hasta por varias horas, luego de que se exponen a la luz o a radiaciones energéticas como la beta. Utilizando este principio se fabrican pigmentos que "alumbran en la oscuridad", como avisos de salida y marcas para indicar caminos (Figura 6).
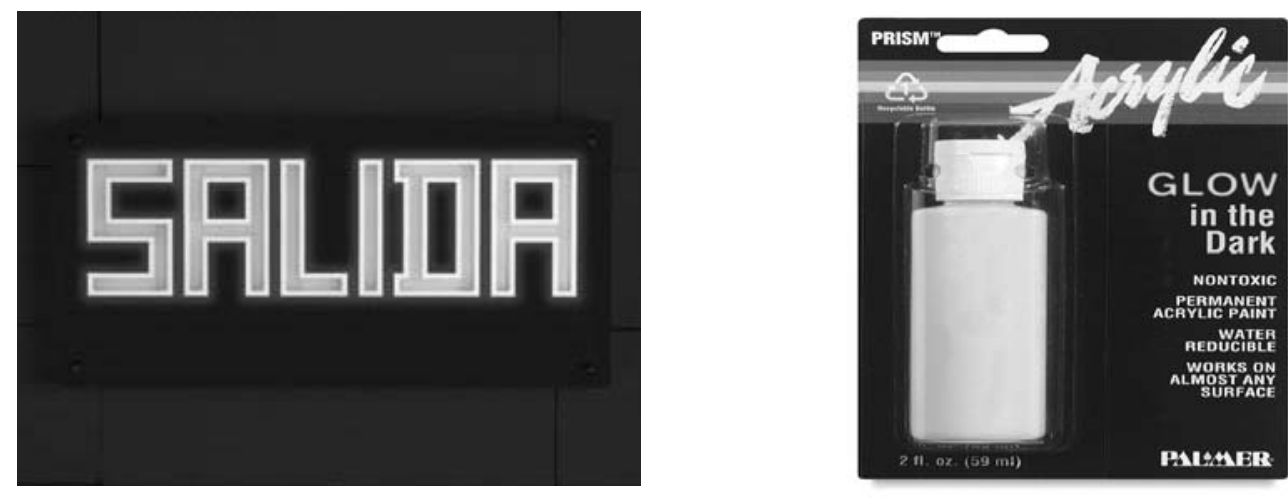

Figura 6. Productos que emiten luz permanentemente 
Los materiales fosforescentes son compuestos químicos de diverso tipo: óxidos, sulfuros o silicatos de elementos cuyos átomos presentan la propiedad de la fosforescencia, que corresponden a elementos conocidos como de transición (Número Atómico 21 a 30, 39 a 48, 57, 72 a 80, 89 y 104 a 112) por ejemplo Zinc, Cobre, Cadmio o Manganeso, o Tierras Raras (Número Atómico 58 a 71 y 90 a 103).

Se han desarrollado diversos compuestos fosforescentes para emitir luz de diferentes colores. Por ejemplo en los tubos de rayos Catódicos de las antiguas televisiones, los electrones que llegan sobre los materiales fosforescentes depositados en el interior de la pantalla del tubo dan origen a la emisión de diferentes colores: el Sulfuro de Zinc activado con Cobre y Aluminio (ZnS:Cu,Al) produce luz verde; el Sulfuro de Zinc activado con Plata (ZnS:Ag) emite luz de color azul; el Sulfuro de Cadmio y Zinc activado con Plata emite luz amarilla; el Oxido-Sulfuro de Itrio activado con Europio $\left(\mathrm{Y}_{2} \mathrm{O}_{2} \mathrm{~S}\right.$ :Eu) produce luz roja.

El principio de la fosforescencia se utilizó el siglo pasado para pintar instrumentos y para los números y agujas en los relojes de muñeca con el fin de hacerlos luminosos. Para ello se mezclaban Sulfuro de Zinc activado con Plata (ZnS:Ag) y materiales radiactivos emisores de radiación alfa o beta, donde el material fosforescente era excitado por la radiación. Esta mezcla emite color verde. Si se requería la emisión de luz azulosa, se mezclaba el material radiactivo con Sulfuro de Zinc activado con Cobre (ZnS:Cu). La luz amarilla se obtenía a partir de mezclar el material radiactivo con Sulfuro de Zinc que contenía Magnesio y Cobre (ZnS:Mg,Cu).

\subsection{LUCES DE TRITIO}

Con el objeto de obtener visibilidad en la oscuridad se fabrican luces de Tritio. Para ello se llenan con gas de Tritio, cápsulas o recipientes de vidrio de alta resistencia que han sido recubiertos en su interior por un material fosforescente. De esta manera se fabrican diminutas luces para colocar en relojes haciendo visibles las manecillas y los números, luces para colocar en las miras nocturnas de armas y otros elementos tácticos, luces de diversa forma para hacer luminosos los avisos de emergencia en industrias y sitios públicos.

Teniendo en cuenta que el Tritio es un elemento radiactivo que se utiliza para propósitos bélicos, son pocas las empresas que lo fabrican y un número reducido lo utilizan para hacer luces de Tritio. El costo del Tritio es alto, aproximadamente USD2 por 37 GBq.

Como se muestra en la Figura 7 se fabrican luces de Tritio que emiten radiación ultravioleta o infrarroja la cual es visible para visores nocturnos especiales. Estas luces son útiles para marcar rutas o elementos que no son visibles a simple vista en la noche, permitiendo distinguir elementos amigos y enemigos más fácilmente. 


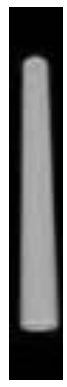

Verde

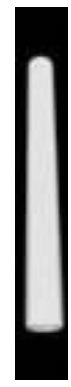

Amarillo

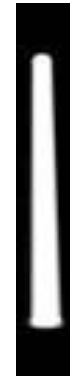

Blanco

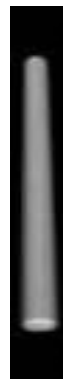

Azul

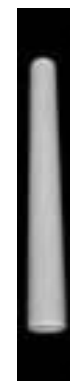

Naranja
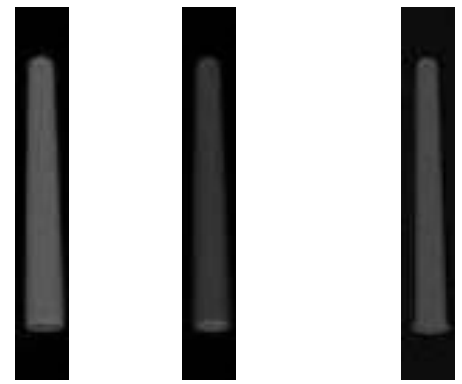

Figura 7. Luces de Tritio

Para obtener diversos colores (Figura 7) se emplean diferentes materiales fosforescentes que son excitados por la radiación beta (electrones) emitidos por el Tritio (Figura 8). Se prefiere emplear este elemento radiactivo y no otros, debido a la baja energía de los electrones emitidos, que hace que la manipulación de los elementos de vidrio donde va el Tritio, no sea riesgosa para las personas.

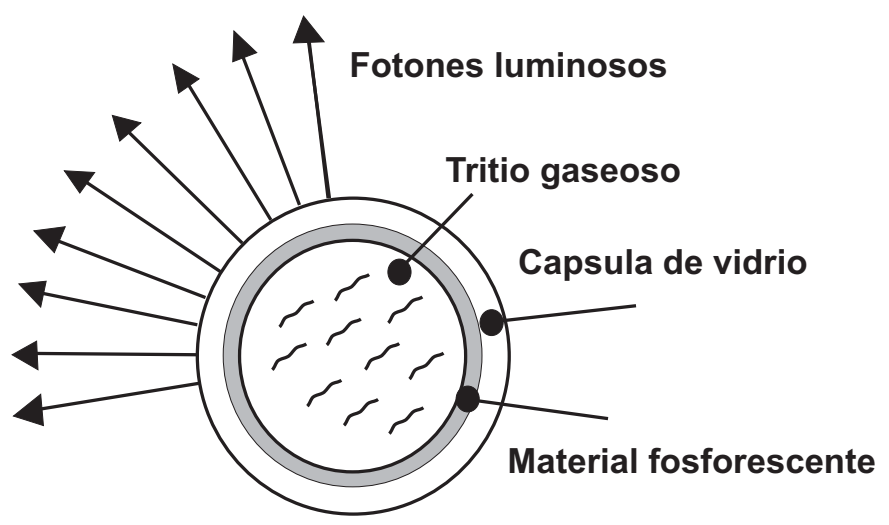

Figura 8. Mecanismo de producción de luz
Para las miras nocturnas de los fusiles Galil, las luces de Tritio [4] miden pocos milímetros. En ellas la luminosidad inicial depende de varios factores como: presión del gas en la cápsula, forma de la cápsula, cantidad de Tritio inyectado, impurezas en el gas y el espesor del material fosforescente.

Los materiales fosforescentes que emiten luz verde son siempre más brillantes que los que emiten otros colores. Para aumentar la luminosidad es usual pintar externamente y de manera parcial la cápsula con pintura reflectiva.

Teniendo en cuenta la Vida media del Tritio, 12,26 años, la luminosidad de la luz de Tritio va disminuyendo con el tiempo, aproximadamente $10 \%$ por año. En el caso de las miras nocturnas, esta se ve así mismo afectada por el material fosforescente usado, las fugas de Tritio a través de imperfecciones de la cápsula, etc. La Figura 9 muestra cómo el brillo decae con el tiempo para diferentes luces de Tritio. Es normal que las cápsulas se fabriquen para durar un periodo de 10 a 20 años, lo que implica que en cada cápsula se inyecte poco menos de un GBq. 


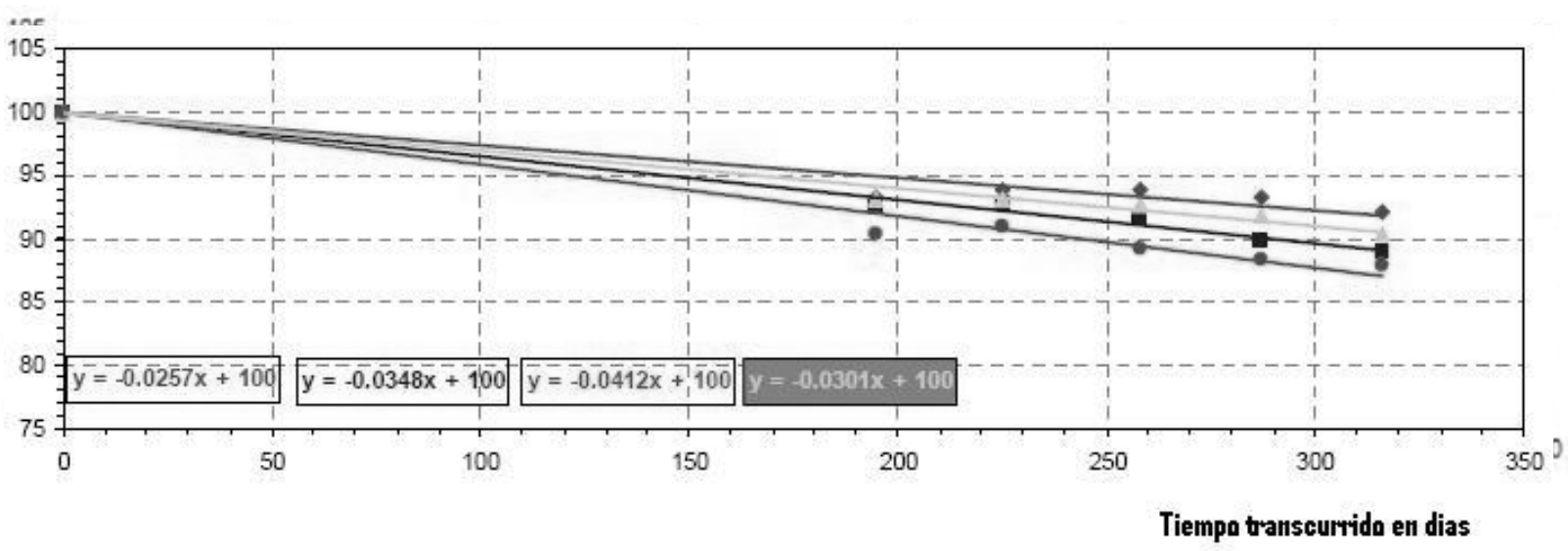

Figura 9. Reducción en luminosidad para cápsulas de Tritio [6].

\section{CONCLUSIONES}

La investigación realizada muestra como es que las luces de Tritio de las miras nocturnas de los fusiles GALIL fabricados por INDUMIL emiten luz verde en la oscuridad. Además, que se pueden utilizar luces de Tritio emisoras de otros colores para la fabricación de otros elementos tácticos tales como lectores de mapas, compases, identificadores de armas, etc. Así mismo existen luces de Tritio que no emiten luz visible sino radiaciones infrarrojas y ultravioleta, emisiones que pueden ser detectadas por visores especiales; estas luces no pueden ser vistas por nuestros ojos por lo que tienen interesantes aplicaciones militares.

En el futuro, INDUMIL desea diversificar su producción para fabricar elementos de uso civil. En este caso las luces de Tritio se pueden usar para hacer visibles los avisos de salida, señalización de emergencia, marcadores para caminos de evacuación en aviones, etc. sin necesidad de usar electricidad.

\section{REFERENCIAS BIBLIOGRAFICAS}

[1] EMERY, Bob; From Quanta to Quarka; Physics HSC Course; http://webs.mn.catholic. edu.au/physics/emery/assets/9 5 op13.gif

[2] FUSIL GALIL 5.56. INDUMIL Colombia. http://www.indumil.gov.co/frameCategoria. asp?ldCat $=16 \&$ IdCatmostrar $=16$

[3] MODELO ATÔMICO DE BOHR. http://es.wikipedia.org/wiki/Modelo at\%C3\% B3mico de Bohr 
[4] SELF ACTIVATED ILLUMINATION; mb-Microtec; Schweiz; http://www.mbmicrotec. $\underline{\mathrm{com} /}$

[5] SOLDIER OF FORTUNE MAGAZINE. 1983. The Arms Site. http://www.remtek.com/ arms/imi/galil/index.htm

[6] TECHNICAL NOTE: T1001. Rate of decay of Glowell ${ }^{\mathrm{TM}}$ light output over time. GLOWELL.

[7] ZUMDAHL, Steven; Introductory Chemistry: A Foundation; $5^{\text {th }}$ Edition; University of Illinois; Urbana-Champaign; 2004. 